\title{
Structural Matrix Analysis of Variable Curvature Curved Beam Dong Changjun ${ }^{1}$ a

\author{
${ }^{1}$ School of Civil Engineering, Lanzhou Jiaotong University, Lanzhou, Gansu, China 730070 \\ aE-mail: Donchangjun.1979@163.com
}

\begin{abstract}
Keyword: Element Stiffness Matrix, Variable Curvature Curved Beam, Element Flexibility Matrix, Equivalent Node Loads

Abstract. In this paper, the finite element analysis of a special form of variable curvature curved beam element is studied, and equilibrium equations of variable curvature curved beam element are established in polar coordinates. Based on the principle of energy method, the relationship between nodes displacement and force is obtained and expressed in the form of matrix. By matrix integral operation, the variable curvature curved beam element flexibility matrix is obtained and is expressed in the form of analytic solution. By the element flexibility matrix inversion, the element stiffness matrix is obtained. The research achievement in this paper provides a new a kind of method in the research of finite element analysis of variable curvature curved beam. The finite element calculation of constant curvature curve beam is only a special case of the calculation method in this paper.
\end{abstract}

\section{Introduction}

Curved girder is widely used in bridge construction because it can adapt to the limit of terrain and object well, but as the bridge construction is restricted by the topography, landforms, or other factors, different curvature bridges need to be decorated together and meanwhile variable curvature curve beam is required as a transition between different curvature beams. And furthermore variable curvature curve beam can also be used as a transition between curve beam and straight beam. Since the early 80 s in last century, a large number of scholars have made theoretical research and experimental study on curved girder. And fruitful results have been achieved ${ }^{[1-8]}$. Based on the energy variation principle ${ }^{[9]}$ and using elastic kernel method, Professor Wu Hongqing and other scholars ${ }^{[10-13]}$ have obtained the curved beam element stiffness matrix which can avoid the introduction of the shape function and the solution of the differential equation. In this paper, on the basis of energy method, analytic solution of the special variable curvature curved beam element stiffness matrix is deduced and the method of putting the load in the middle of unit into the equivalent nodal load is obtained.

In this paper the following assumptions are made. Firstly, the cross section centroid and shear centre is coincidence or close to overlap. Secondly, the cross section size of curved beam is much smaller than the radius of curvature.

\section{Unit flexibility matrix of variable curvature curved beam}

As shown in Figure 1, there is a variable curvature curve beam. Beam axis equation in local polar coordinates can be expressed as

$$
\rho=\rho(\alpha)
$$

According to the right-hand rule, the floating coordinate system is set up at each point on the axis shown in Figure 1. Among them, the $\xi$ axis is located in the tangent direction of the axis, the $\eta$ axis is located in the radial direction of the axis and $\zeta$ axis is perpendicular to the plane in which there is the axis of curved beam. The point $i$ and point $j$ is the ends of curved beam element and point $M$ whose coordinates is $\xi \eta \zeta$ is any point on the curved beam. Firstly, several concepts are defined. The Central Angle $\theta_{0}$ is angle of point $i$ radius of curvature extension and point $j$ radius of curvature extension. Angle $\theta$ is angle of point $M$ radius of curvature extension and 
point $j$ radius of curvature extension. According to the geometric relationship, Angle $\theta$ is equal to the angle of tangent at point $M$ and point $j$. The distance between point $M$ and point $j$ radius of curvature is $L_{2}$. The distance between point $M$ and point $j$ tangent is $L_{1}$. The distance between point $j$ and point $M$ radius of curvature is $L_{4}$. The distance between point $j$ and point $M$ tangent is $L_{3}$. The polar angle of point $j$ is $\theta_{1}$ in local coordinate system and the polar angle of point $M$ is $\alpha$. The difference between polar angle of point $M$ and polar angle of point $j$ is $\beta$.

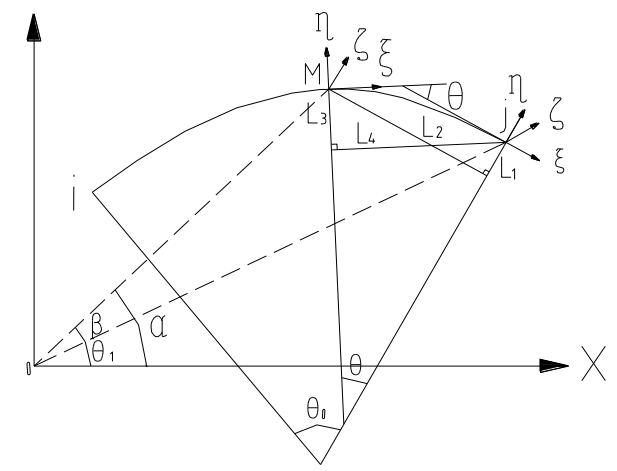

Fig. 1 Variable curvature curved beam element and coordinate system

The three linear displacements at node $i$ are tangential displacement $u_{i \xi}$, radial displacement $v_{i n}$ and horizontal displacement $w_{i \zeta}$. The three angular displacements at node $i$ are torsion angle $\theta_{i \xi}$, bending angle $\theta_{i n}$ and bending angle $\theta_{i \zeta}$. Corresponding node forces are axial force $N_{i \xi}$, radial shear $Q_{i \eta}$, transverse shear $Q_{i \xi}$, torque $M_{i \xi}$, bending moment $M_{i n}$ and bending moment $M_{i \xi}$. The displacements and node forces at node $j$ are similar to them at node $i$. The positive direction of the displacements and node forces is equal to the positive direction of local coordinate system at the node.

The angle between polar axis and the curve tangent is $\gamma$. There is equation can be expressed as

$$
\gamma=\arctan \frac{\rho(\alpha)}{\rho^{\prime}(\alpha)} .
$$

(2)

According to geometry relationship in Figure 1, the equation can be expressed as

$$
\beta=\theta+\arctan \frac{\rho(\alpha)}{\rho^{\prime}(\alpha)}-\arctan \frac{\rho\left(\theta_{1}\right)}{\rho^{\prime}\left(\theta_{1}\right)} .
$$

The slope at any point in the curve can be described as

$$
k=\frac{\rho^{\prime} \sin \alpha+\rho \cos \alpha}{\rho^{\prime} \cos \alpha-\rho \sin \alpha} .
$$

(4)

According to the distance formula between point and straight line, the formula (5) is established.$$
L_{1}= \pm L_{i}^{\prime}= \pm \frac{-\rho(\alpha) \frac{\rho^{\prime}\left(\theta_{1}\right)}{\rho\left(\theta_{1}\right)} \sin \left(\alpha-\theta_{1}\right)+\rho(\alpha) \cos \left(\alpha-\theta_{1}\right)-\rho\left(\theta_{1}\right)}{\sqrt{1+\frac{\rho^{\prime}\left(\theta_{1}\right)^{2}}{\rho\left(\theta_{1}\right)^{2}}}} .
$$

If the pole and the point $M$ are on the same side of the tangent at point $j, "+"$ is taken in the above formula. If the pole and the point $M$ are on the different sides of the tangent at point $j$, , $-"$ is taken in the above formula.

In a similar way, 


$$
L_{2}= \pm L_{2}^{\prime}= \pm \frac{\rho(\alpha) \frac{\rho\left(\theta_{1}\right)}{\rho^{\prime}\left(\theta_{1}\right)} \sin \left(\alpha-\theta_{1}\right)+\rho(\alpha) \cos \left(\alpha-\theta_{1}\right)-\rho\left(\theta_{1}\right)}{\sqrt{1+\frac{\rho\left(\theta_{1}\right)^{2}}{\rho^{\prime}\left(\theta_{1}\right)^{2}}}} .
$$

(6)

If the pole and point $M$ are on the same side of the tangent at node $j$ and $\rho^{\prime}\left(\theta_{1}\right)>0, "-"$ is taken in the above formula. If the pole and point $M$ are on the different sides of the tangent at node $j$ and $\rho^{\prime}\left(\theta_{1}\right)>0$, "+" is taken in the above formula. If $\rho^{\prime}\left(\theta_{1}\right)=0$ and $\sin \left(\alpha-\theta_{1}\right) \geq 0$, "+" is taken in the above formula. If $\rho^{\prime}\left(\theta_{1}\right)=0$ and $\sin \left(\alpha-\theta_{1}\right)<0, "-"$ is taken in the above formula. If the pole and point $M$ are on the same side of the tangent at node $j$ and $\rho^{\prime}\left(\theta_{1}\right)<0, "+"$ is taken in the above formula. If the pole and point $M$ are on the different sides of the tangent at node $j$ and $\rho^{\prime}\left(\theta_{1}\right)<0$, "-" is taken in the above formula.

$$
L_{3}= \pm L_{3}^{\prime}= \pm \frac{-\rho\left(\theta_{1}\right) \frac{\rho^{\prime}(\alpha)}{\rho(\alpha)} \sin \left(\alpha-\theta_{1}\right)+\rho\left(\theta_{1}\right) \cos \left(\alpha-\theta_{1}\right)-\rho(\alpha)}{\sqrt{1+\frac{\rho^{\prime}(\alpha)^{2}}{\rho(\alpha)^{2}}}} .
$$

If the pole and node $j$ are on the same side of the tangent at point $M, "-"$ is taken in the above formula. If the pole and node $j$ are on the different sides of the tangent at point $M, \quad "+$ " is taken in the above formula.

$$
L_{4}= \pm L_{4}^{\prime}= \pm \frac{\rho\left(\theta_{1}\right) \frac{\rho(\alpha)}{\rho^{\prime}(\alpha)} \sin \left(\alpha-\theta_{1}\right)+\rho\left(\theta_{1}\right) \cos \left(\alpha-\theta_{1}\right)-\rho(\alpha)}{\sqrt{1+\frac{\rho(\alpha)^{2}}{\rho^{\prime}(\alpha)^{2}}}} .
$$

If the pole and node $j$ are on the same side of the radius of curvature at point $M$ and $\rho^{\prime}(\alpha)>0$, "-" is taken in the above formula. If the pole and node $j$ are on the different sides of the radius of curvature at point $M$ and $\rho^{\prime}(\alpha)>0$, " + " is taken in the above formula. If $\rho^{\prime}(\alpha)=0$ and $\sin \left(\alpha-\theta_{1}\right) \geq 0$, " $-"$ is taken in the above formula. If $\rho^{\prime}(\alpha)=0$ and $\sin \left(\alpha-\theta_{1}\right)<0, \quad "+"$ is taken in the above formula. If the pole and node $j$ are on the same side of the radius of curvature at point $M$ and $\rho^{\prime}(\alpha)<0$, "+" is taken in the above formula. If the pole and node $j$ are on the different sides of the radius of curvature at point $M$ and $\rho^{\prime}(\alpha)<0$, "-" is taken in the above formula.

Assuming that $i$ end is fixed end of the curved beam element and according to equilibrium equation, the internal force at any point in the curved beam can be expressed as

$$
\left\{\begin{array}{l}
N_{\xi}=-N_{j \xi} \cos \theta-Q_{j n} \sin \theta \\
Q_{n}=N_{j s} \sin \theta-Q_{j \eta} \cos \theta \\
Q_{\xi}=-Q_{j 5}
\end{array}\right.
$$

$$
\begin{aligned}
& M_{\xi}=M_{\xi}= \pm Q_{j \xi} L_{3}-M_{j \xi} \cos \theta-M_{j \eta} \sin \theta
\end{aligned}
$$

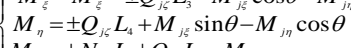

If the pole and node $j$ are on the same side of the tangent at point $M$, "+" is taken in the formula(10). If the pole and the point $M$ are on the different sides of the tangent at point $j$, , " is taken in the above formula(10). So,

$$
\left\{\begin{array}{l}
M_{\xi}=M_{\xi}=-Q_{j \zeta} L_{3}^{\prime}-M_{j \xi} \cos \theta-M_{j \eta} \sin \theta \\
M_{\eta}=-Q_{j \zeta} L_{4}^{\prime}+M_{j \xi} \sin \theta-M_{j \eta} \cos \theta \\
M_{\zeta}=-N_{j \xi} L_{1}^{\prime}-Q_{j \eta} L_{2}^{\prime}-M_{j \zeta}
\end{array}\right.
$$

(11)

Strain energy in the beam can be expressed as

$$
\begin{aligned}
\Pi= & \frac{1}{2} \int_{s} \frac{\left(-N_{j \xi} \cos \theta-Q_{j \eta} \sin \theta\right)^{2}}{E A_{\xi}} d s+\frac{1}{2} \int \frac{\left(N_{j \xi} \sin \theta-Q_{j \eta} \cos \theta\right)^{2}}{G A_{\eta}} d s+\frac{1}{2} \int_{s} \frac{Q_{j \xi}^{2}}{G A_{\zeta}} d s+\frac{1}{2} \int_{s} \frac{\left(-Q_{j \xi} L_{3}^{\prime}-M_{j \xi} \cos \theta-M_{i n} \sin \theta\right)^{2}}{G I_{\xi}} d s . \\
& +\frac{1}{2} \int_{s} \frac{\left(-Q_{j \xi} L_{4}^{\prime}+M_{j \xi} \sin \theta-M_{j n} \cos \theta\right)^{2}}{E I_{\eta}} d s+\frac{1}{2} \int_{s} \frac{\left(-N_{j \xi} L_{1}^{\prime}-Q_{j \eta} L_{2}^{\prime}-M_{j \xi}\right)^{2}}{E I_{\zeta}} d s
\end{aligned} .
$$


(12)

According to Castigliano second theorem, the formulas (13-18) are established.

$$
\begin{aligned}
u_{j \xi} & =\frac{\partial \Pi}{\partial N_{j \xi}}=\int_{s} \frac{\left(-N_{j \xi} \cos \theta-Q_{i n} \sin \theta\right)(-\cos \theta)}{E A_{\xi}} d s+\int_{s} \frac{\left(N_{j \xi} \sin \theta-Q_{i n} \cos \theta\right) \sin \theta}{G A_{\eta}} d s+\int_{s} \frac{\left(-N_{j \xi} L_{1}^{\prime}-Q_{j \eta} L_{2}^{\prime}-M_{j \xi}\right)\left(-L_{1}^{\prime}\right)}{E I_{\zeta}} d s \\
& =\int_{s} \frac{N_{j \xi} \cos ^{2} \theta}{E A_{\xi}} d s+\int_{s} \frac{Q_{j n} \sin \theta \cos \theta}{E A_{\xi}} d s+\int_{s} \frac{N_{j \xi} L_{1}^{n}}{E I_{\zeta}} d s+\int_{s} \frac{Q_{j n} L_{1}^{\prime} L_{2}^{\prime}}{E I_{\zeta}} d s+\int \frac{M_{j \xi} L_{i}^{\prime}}{E I_{\zeta}} d s+\int_{s} \frac{N_{j \xi} \sin ^{2} \theta}{G A_{\eta}} d s-\int \frac{Q_{j \eta} \sin \theta \cos \theta}{G A_{\eta}} d s
\end{aligned}
$$

$$
\begin{aligned}
v_{j \eta} & =\frac{\partial \Pi}{\partial N_{j \eta}}=\int_{s} \frac{\left(-N_{j \xi} \cos \theta-Q_{j \eta} \sin \theta\right)(-\sin \theta)}{E A_{\xi}} d s+\int_{s} \frac{\left(N_{j \xi} \sin \theta-Q_{j \eta} \cos \theta\right)(-\cos \theta)}{G A_{\eta}} d s+\int_{s} \frac{\left(-N_{j \xi} L_{1}^{\prime}-Q_{j \eta} L_{2}^{\prime}-M_{j \xi}\right)\left(-L_{2}^{\prime}\right)}{E I_{\zeta}} d s ; \\
& =\int_{s} \frac{N_{j \xi} \sin \theta \cos \theta}{E A_{\xi}} d s+\int_{s} \frac{Q_{j \eta} \sin ^{2} \theta}{E A_{\xi}} d s+\int_{s} \frac{N_{j \xi} L_{1}^{\prime} L_{2}^{\prime}}{E I_{\zeta}} d s+\int_{s} \frac{Q_{j n} L_{2}^{\prime 2}}{E I_{\zeta}} d s+\int_{s} \frac{M_{j \xi} L_{2}^{\prime}}{E I_{\zeta}} d s-\int_{s} \frac{N_{j \xi} \sin \theta \cos \theta}{G A_{\eta}} d s+\int_{s} \frac{Q_{j \eta} \cos ^{2} \theta}{G A_{\eta}} d s
\end{aligned}
$$

$$
\begin{aligned}
w_{j \xi} & =\frac{\partial \Pi}{\partial N_{j \xi}}=\int_{s} \frac{Q_{j \xi}}{G A_{\zeta}} d s+\int_{s} \frac{\left(-Q_{j \xi} L_{3}^{\prime}-M_{j \xi} \cos \theta-M_{j \eta} \sin \theta\right)\left(-L_{3}^{\prime}\right)}{G I_{\xi}} d s+\frac{1}{2} \int_{s} \frac{\left(-Q_{i \xi} L_{4}^{\prime}+M_{j \xi} \sin \theta-M_{j \eta} \cos \theta\right)\left(-L_{4}^{\prime}\right)}{E I_{\eta}} d s \\
& =\int_{s} \frac{Q_{j \xi} L_{3}^{2}}{G I_{\xi}} d s+\int_{s} \frac{M_{j \xi} L_{3}^{\prime} \cos \theta}{G I_{\xi}} d s+\int_{s} \frac{M_{j n} L_{3}^{\prime} \sin \theta}{G I_{\xi}} d s+\int_{s} \frac{Q_{j \xi} L_{4}^{2}}{E I_{\eta}} d s-\int_{s} \frac{M_{j \xi} L_{4}^{\prime} \sin \theta}{E I_{\eta}} d s+\int_{s} \frac{M_{j \eta} L_{4}^{\prime} \cos \theta}{E I_{\eta}} d s+\int_{s} \frac{Q_{j \zeta}}{G A_{\xi}} d s
\end{aligned}
$$

$$
\begin{aligned}
\theta_{j \xi} & =\frac{\partial \Pi}{\partial M_{j \xi}}=\int_{s} \frac{\left(-Q_{j \xi} L_{3}^{\prime}-M_{j \xi} \cos \theta-M_{j \eta} \sin \theta\right)(-\cos \theta)}{G I_{\xi}} d s+\int_{s} \frac{\left(-Q_{j \xi} L_{4}^{\prime}+M_{j \xi} \sin \theta-M_{i n} \cos \theta\right) \sin \theta}{E I_{\eta}} d s \\
& =\int_{s} \frac{Q_{j \xi} L_{3}^{\prime} \cos \theta}{G I_{\xi}} d s+\int_{s} \frac{M_{j \xi} \cos ^{2} \theta}{G I_{\xi}} d s+\int_{s} \frac{M_{j \eta} \sin \theta \cos \theta}{G I_{\xi}} d s-\int_{s} \frac{Q_{j \xi} L_{4}^{\prime} \sin \theta}{E I_{\eta}} d s+\int_{s} \frac{M_{j \xi} \sin ^{2} \theta}{E I_{\eta}} d s-\int_{s} \frac{M_{j \eta} \sin \theta \cos \theta}{E I_{\eta}} d s
\end{aligned}
$$

$$
\begin{aligned}
\theta_{j \eta} & =\frac{\partial \Pi}{\partial M_{j \eta}}=\int \frac{\left(-Q_{j \xi} L_{3}^{\prime}-M_{j \xi} \cos \theta-M_{j \eta} \sin \theta\right)(-\sin \theta)}{G I_{\xi}} d s+\int_{s} \frac{\left(-Q_{j \xi} L_{4}^{\prime}+M_{j \xi} \sin \theta-M_{j \eta} \cos \theta\right)(-\cos \theta)}{E I_{\eta}} d s \\
& =\int_{s} \frac{Q_{j \xi} L_{3}^{\prime} \sin \theta}{G I_{\xi}} d s+\int_{s} \frac{M_{j \xi} \sin \theta \cos \theta}{G I_{\xi}} d s+\int_{s} \frac{M_{j \eta} \sin ^{2} \theta}{G I_{\xi}} d s+\int_{s} \frac{Q_{j \xi} L_{4}^{\prime} \cos \theta}{E I_{\eta}} d s-\int_{s} \frac{M_{j \xi} \sin \theta \cos \theta}{E I_{\eta}} d s+\int_{s} \frac{M_{i n} \cos ^{2} \theta}{E I_{\eta}} d s
\end{aligned}
$$$$
\theta_{j \zeta}=\frac{\partial \Pi}{\partial M_{j \zeta}}=\int_{s} \frac{\left(-N_{j \xi} L_{1}^{\prime}-Q_{i n} L_{2}^{\prime}-M_{j \zeta}\right)(-1)}{E I_{\zeta}} d s=\int_{s} \frac{N_{j i} L_{1}^{\prime}}{E I_{\zeta}} d s+\int_{s_{i}} \frac{Q_{i n} L_{2}^{\prime}}{E I_{\zeta}} d s+\int_{s} \frac{M_{j \zeta}}{E I_{\zeta}} d s
$$

The above formulas can be written in matrix form.

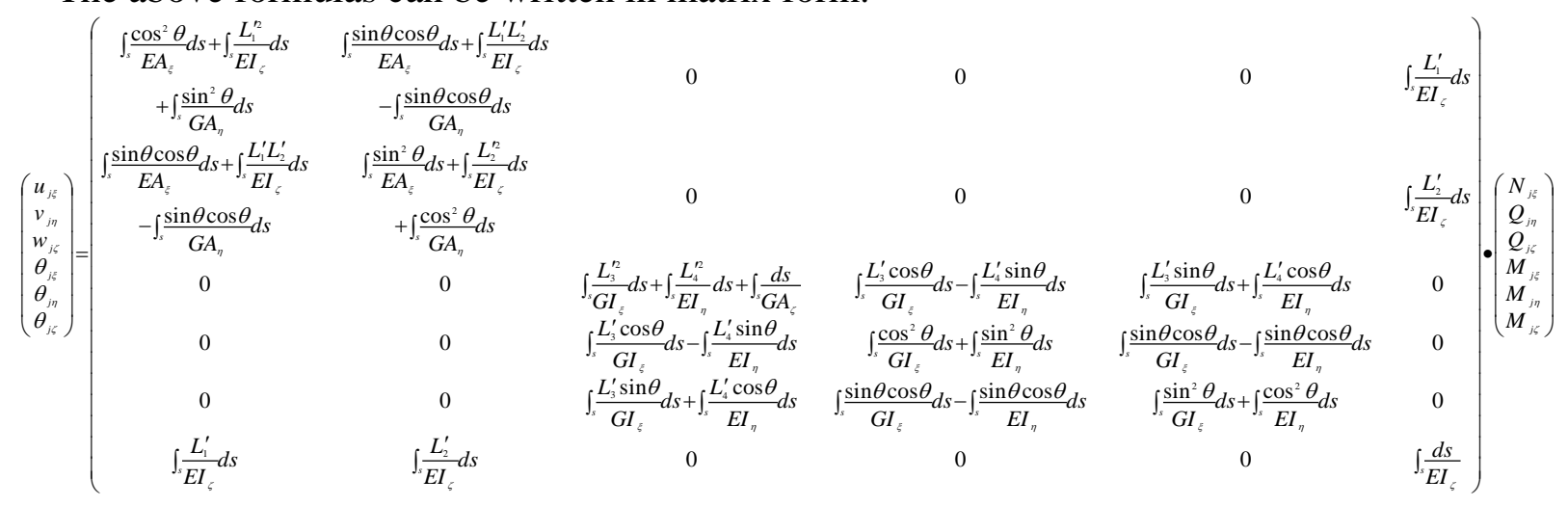

(19)

It also can be expressed as

$\delta_{j}^{e}=f F_{j}^{e}$.

(20)

If axis equation of curved beam in local polar coordinates can be expressed as $\rho=a e^{b \alpha}(a>0)$.

$\theta=\beta=\alpha-\theta_{1}$. 
(22)

Each element of matrix in integral operation, the results are got.

The radius of curvature at point $j$ can be expressed as

$R_{j}=a \sqrt{1+b^{2}} e^{b \theta_{1}}$.

(23)

So,

$$
\begin{aligned}
& f_{11}=\frac{R_{j}}{E A_{\xi}}\left[\frac{\mathrm{e}^{b \theta_{0}}-1}{2 b}+\frac{2 \mathrm{e}^{b \theta_{0}} \sin 2 \theta_{0}+b\left(\mathrm{e}^{b \theta_{0}} \cos 2 \theta_{0}-1\right)}{2\left(4+b^{2}\right)}\right]+\frac{R_{j}}{G A_{n}}\left[\frac{\mathrm{e}^{b \theta_{0}}-1}{2 b}-\frac{2 \mathrm{e}^{b \theta_{0}} \sin 2 \theta_{0}+b\left(\mathrm{e}^{b \theta_{0}} \cos 2 \theta_{0}-1\right)}{2\left(4+b^{2}\right)}\right] \\
& +\frac{R_{j}^{3}}{E I_{\zeta}\left(1+b^{2}\right)^{2}}\left[\frac{\left(\mathrm{e}^{3 b \theta_{0}}-1\right)\left(1+b^{2}\right)}{6 b}+\frac{\mathrm{e}^{b \theta_{0}}-1}{b}+\frac{\left(2-8 b^{2}\right) \mathrm{e}^{3 b \theta_{0}} \sin 2 \theta_{0}+\left(7 b-3 b^{3}\right)\left(\mathrm{e}^{3 b \theta_{0}} \cos 2 \theta_{0}-1\right)}{2\left(4+9 b^{2}\right)}+\frac{\left(4 b^{2}-2\right) \mathrm{e}^{2 b \theta_{0}} \sin \theta_{0}-6 b\left(\mathrm{e}^{2 b \theta_{0}} \cos \theta_{0}-1\right)}{1+4 b^{2}}\right] \\
& f_{12}=\int_{s} \frac{\sin \theta \cos \theta}{E A_{\xi}} d s+\int_{s} \frac{L_{1}^{\prime} L_{2}^{\prime}}{E I_{\zeta}} d s-\int \frac{\sin \theta \cos \theta}{G A_{\eta}} d s=\frac{R_{j}}{2 E A_{\xi}}\left[\frac{e^{b \theta_{0}}\left(b \sin 2 \theta_{0}-2 \cos 2 \theta_{0}\right)+2}{4+b^{2}}\right]-\frac{R_{j}}{2 G A_{\eta}}\left[\frac{e^{b \theta_{0}}\left(b \sin 2 \theta_{0}-2 \cos 2 \theta_{0}\right)+2}{4+b^{2}}\right] \text {; } \\
& +\frac{R_{j}^{3}}{E I_{\zeta}\left(1+b^{2}\right)^{2}}\left[\frac{\left(7 b-3 b^{3}\right) e^{3 b \theta_{0}} \sin 2 \theta_{0}+\left(8 b^{2}-2\right)\left(e^{3 b \theta_{0}} \cos 2 \theta_{0}-1\right)}{2\left(4+9 b^{2}\right)}+\frac{\left(2 b^{3}-4 b\right) e^{2 b \theta_{0}} \sin \theta_{0}+\left(5 b^{2}-1\right)\left(e^{2 b \theta_{0}} \cos \theta_{0}-1\right)}{1+4 b^{2}}+e^{b \theta_{0}}-1\right] \\
& f_{16}=\int_{s} \frac{L_{1}^{\prime}}{E I_{\zeta}} d s=\frac{R_{j}^{2}}{E I_{\zeta}\left(1+b^{2}\right)}\left[-\frac{e^{b \theta_{0}}-1}{b}+\frac{\left(1-2 b^{2}\right) e^{2 b \theta_{0}} \sin \theta_{0}+3 b\left(e^{2 b \theta_{0}} \cos \theta_{0}-1\right)}{1+4 b^{2}}\right] ; \\
& f_{22}=\int_{s} \frac{\sin ^{2} \theta}{E A_{\xi}} d s+\int_{s} \frac{L_{2}^{2}}{E I_{\zeta}} d s+\int_{s} \frac{\cos ^{2} \theta}{G A_{\eta}} d s=\frac{R_{j}}{E A_{\xi}}\left[\frac{e^{b \theta_{0}}-1}{2 b}-\frac{2 e^{b \theta_{0}} \sin 2 \theta_{0}+b\left(e^{b \theta_{0}} \cos 2 \theta_{0}-1\right)}{2(4+b)}\right]+\frac{R_{j}}{G A_{\eta}}\left[\frac{e^{b \theta_{0}}-1}{2 b}+\frac{2 e^{b \theta_{0}} \sin 2 \theta_{0}+b\left(e^{b \theta_{0}} \cos 2 \theta_{0}-1\right)}{2(4+b)}\right] \\
& +\frac{R_{j}^{3}}{E I_{\zeta}\left(1+b^{2}\right)^{2}}\left[\frac{\left(e^{3 b \theta_{0}}-1\right)\left(1+b^{2}\right)}{6 b}+b\left(e^{b \theta_{0}}-1\right)-\frac{\left(2-8 b^{2}\right) e^{3 b \theta_{0}} \sin 2 \theta_{0}+\left(7 b-3 b^{3}\right)\left(e^{3 b b_{0}} \cos 2 \theta_{0}-1\right)}{2\left(4+9 b^{2}\right)}+\frac{6 b^{2} e^{2 b \theta_{0}} \sin \theta_{0}+\left(4 b^{3}-2 b\right)\left(e^{2 b \theta_{0}} \cos \theta_{0}-1\right)}{1+4 b^{2}}\right] \\
& f_{26}=\int_{s} \frac{L_{2}^{\prime}}{E I_{\zeta}} d s=\frac{R_{j}^{2}}{E I_{\zeta}\left(1+b^{2}\right)}\left[\frac{3 b e^{2 b \theta_{0}} \sin \theta_{0}+\left(2 b^{2}-1\right)\left(e^{2 b \theta_{0}} \cos \theta_{0}-1\right)}{1+4 b^{2}}-e^{b \theta_{0}}+1\right] \text {; } \\
& f_{33}=\int_{s} \frac{L_{3}^{2}}{G I_{\xi}} d s+\int_{s}^{\frac{L_{4}^{2}}{E I_{\eta}}} d s+\int_{s} \frac{d s}{G A_{\zeta}} \\
& =\frac{R_{j}}{b G A_{\xi}}\left(e^{b \theta_{0}}-1\right)+\frac{R_{j}^{3}}{G I_{\xi}\left(1+b^{2}\right)^{2}}\left[\frac{\left(e^{b \theta_{0}}-1\right)\left(b^{2}+1\right)}{2 b}+\frac{2 e^{b \theta_{0}} \sin 2 \theta_{0}-\left(b^{3}+3 b\right)\left(e^{b \theta_{0}} \cos 2 \theta_{0}-1\right)}{2\left(4+b^{2}\right)}+\frac{e^{3 b \theta_{0}}-1}{3 b}-\frac{\left(4 b^{2}+2\right) e^{2 b \theta_{0}} \sin \theta_{0}+2 b\left(e^{2 b \theta_{0}} \cos \theta_{0}-1\right)}{1+4 b^{2}}\right] ; \\
& +\frac{R_{j}^{3}}{E I_{n}\left(1+b^{2}\right)^{2}}\left[\frac{\left(e^{b \theta_{0}}-1\right)\left(1+b^{2}\right)}{2 b}+\frac{b\left(e^{3 b b_{0}}-1\right)}{3}-\frac{2 e^{b \theta_{0}} \sin 2 \theta_{0}-\left(b^{3}+3 b\right)\left(e^{b \theta_{0}} \cos 2 \theta_{0}-1\right)}{2\left(4+b^{2}\right)}+\frac{2 b^{2} e^{2 b \theta_{0}} \sin \theta_{0}-\left(4 b^{3}+2 b\right)\left(e^{2 b \theta_{0}} \cos \theta_{0}-1\right)}{1+4 b^{2}}\right] \\
& f_{34}=\int \frac{L_{3}^{\prime} \cos \theta}{G I_{\xi}} d s-\int_{s} \frac{L_{3}^{\prime} \sin \theta}{E I_{\eta}} d s \\
& =\frac{R_{j}^{2}}{G I_{\xi}\left(1+b^{2}\right)}\left[\frac{\left(b^{2}+2\right) e^{b \theta_{0}} \sin 2 \theta_{0}-b\left(e^{b \theta_{0}} \cos 2 \theta_{0}-1\right)}{2\left(4+b^{2}\right)}+\frac{e^{b \theta_{0}}-1}{2 b}-\frac{e^{2 b \theta_{0}} \sin \theta_{0}+2 b\left(e^{2 b \theta_{0}} \cos \theta_{0}-1\right)}{1+4 b^{2}}\right] ; \\
& -\frac{R_{j}^{2}}{E I_{\eta}\left(1+b^{2}\right)}\left[\frac{\left(b^{2}+2\right) e^{b \theta_{0}} \sin 2 \theta_{0}-b\left(e^{b \theta_{0}} \cos 2 \theta_{0}-1\right)}{2\left(4+b^{2}\right)}-\frac{e^{b \theta_{0}}-1}{2 b}-\frac{2 b^{2} e^{2 b \theta_{0}} \sin \theta_{0}-b\left(e^{2 b \theta_{0}} \cos \theta_{0}-1\right)}{1+4 b^{2}}\right] \\
& f_{35}=\int_{s} \frac{L_{3}^{\prime} \sin \theta}{G I_{\xi}} d s+\int_{s} \frac{L_{3}^{\prime} \cos \theta}{E I_{\eta}} d s \\
& =\frac{R_{j}^{2}}{G I_{\xi}\left(1+b^{2}\right)}\left[\frac{e^{b \theta_{0}}-1}{2}-\frac{b e^{b \theta_{0}} \sin 2 \theta_{0}+\left(b^{2}+2\right)\left(e^{b \theta_{0}} \cos 2 \theta_{0}-1\right)}{2\left(4+b^{2}\right)}-\frac{2 b e^{2 b \theta_{0}} \sin \theta_{0}-\left(e^{2 b \theta_{0}} \cos \theta_{0}-1\right)}{1+4 b^{2}}\right] ; \\
& +\frac{R_{j}^{2}}{E I_{\eta}\left(1+b^{2}\right)}\left[\frac{e^{b \theta_{0}}-1}{2}+\frac{b e^{b \theta_{0}} \sin 2 \theta_{0}+\left(b^{2}+2\right)\left(e^{b \theta_{0}} \cos 2 \theta_{0}-1\right)}{2\left(4+b^{2}\right)}-\frac{b e^{2 b \theta_{0}} \sin \theta_{0}+2 b^{2}\left(e^{2 b \theta_{0}} \cos \theta_{0}-1\right)}{1+4 b^{2}}\right] \\
& f_{44}=\int_{s} \frac{\cos ^{2} \theta}{G I_{\xi}} d s+\int_{s} \frac{\sin ^{2} \theta}{E I_{\eta}} d s=\frac{R_{j}}{G I_{\xi}}\left[\frac{2 e^{b \theta_{0}} \sin 2 \theta_{0}+b\left(e^{b \theta_{0}} \cos 2 \theta_{0}-1\right)}{2\left(4+b^{2}\right)}+\frac{e^{b \theta_{0}}-1}{2 b}\right]+\frac{R_{j}}{E I_{\eta}}\left[\frac{e^{b \theta_{0}}-1}{2 b}-\frac{2 e^{b \theta_{0}} \sin 2 \theta_{0}+b\left(e^{b \theta_{0}} \cos 2 \theta_{0}-1\right)}{2\left(4+b^{2}\right)}\right] ; \\
& f_{45}=\int_{s} \frac{\sin \theta \cos \theta}{G I_{\xi}} d s-\int \frac{\sin \theta \cos \theta}{E I_{\eta}} d s=\frac{R_{j}}{G I_{\xi}}\left[\frac{b e^{b \theta_{0}} \sin 2 \theta_{0}-2\left(e^{b \theta_{0}} \cos 2 \theta_{0}-1\right)}{2\left(4+b^{2}\right)}\right]-\frac{R_{j}}{E I_{\eta}}\left[\frac{b e^{b \theta_{0}} \sin 2 \theta_{0}-2\left(e^{b \theta_{0}} \cos 2 \theta_{0}-1\right)}{2\left(4+b^{2}\right)}\right] ; \\
& f_{55}=\int_{s} \frac{\sin ^{2} \theta}{G I_{\xi}} d s+\int_{s} \frac{\cos ^{2} \theta}{E I_{\eta}} d s=\frac{R_{j}}{G I_{\xi}}\left[\frac{e^{b \theta_{0}}-1}{2 b}-\frac{2 e^{b \theta_{0}} \sin 2 \theta_{0}+b\left(e^{b \theta_{0}} \cos 2 \theta_{0}-1\right)}{2\left(4+b^{2}\right)}\right]+\frac{R_{j}}{E I_{\eta}}\left[\frac{e^{b \theta_{0}}-1}{2 b}+\frac{2 e^{b \theta_{0}} \sin 2 \theta_{0}+b\left(e^{b \theta_{0}} \cos 2 \theta_{0}-1\right)}{2\left(4+b^{2}\right)}\right] ; \\
& f_{66}=\int_{s} \frac{d s}{E I_{\zeta}}=\frac{R_{j}\left(e^{b \theta_{0}}-1\right)}{b E I_{\zeta}} .
\end{aligned}
$$


Flexibility matrix of variable curvature cantilever curved beam is obtained and it can be expressed as

$f=\left[\begin{array}{cccccc}f_{11} & f_{12} & 0 & 0 & 0 & 0 \\ f_{21} & f_{22} & 0 & 0 & 0 & 0 \\ 0 & 0 & f_{33} & f_{34} & f_{35} & 0 \\ 0 & 0 & f_{43} & f_{44} & f_{45} & 0 \\ 0 & 0 & f_{53} & f_{54} & f_{55} & 0 \\ f_{61} & f_{62} & 0 & 0 & 0 & f_{63}\end{array}\right]$.

Also,

(25)

The following gives two different degradations of variable curvature curved beam element.

A) If $b=0$, it can be expressed as $\rho=a$. Variable curvature curved beam degrades into constant curvature curved beam. The coefficients of flexibility matrix degrade into the coefficients which are shown in literature ${ }^{[11]}$

B) Arc length is constant and it can be expressed as

$$
s=\frac{a \sqrt{1+b^{2}}}{b}\left[e^{b\left(\theta_{0}+\theta_{1}\right)}-e^{\left.b b_{1}\right)}\right] \cdot
$$

I ) $b$ is constant and $a \rightarrow \infty, \theta \rightarrow 0$,

So,

$$
\begin{aligned}
& \quad f_{11} \rightarrow \frac{s}{E A_{\xi}}, \quad f_{12} \rightarrow 0, \quad f_{16} \rightarrow 0, \quad f_{22} \rightarrow \frac{s^{3}}{3 E I_{\zeta}}+\frac{s}{G A_{\eta}}, f_{26} \rightarrow \frac{s^{2}}{2 E I_{\zeta}}, f_{33} \rightarrow \frac{s^{3}}{3 E I_{\zeta}}+\frac{s}{G A_{\eta}}, f_{34} \rightarrow 0, f_{44} \rightarrow \frac{s}{G I_{\xi}}, f_{45} \rightarrow 0, f_{55} \rightarrow \frac{s}{E I_{\eta}}, \\
& f_{66} \rightarrow \frac{s}{E I_{\zeta}} \\
& \text { II ) } a \text { is constant and } b \rightarrow \infty, \quad \theta_{0} \rightarrow 0,
\end{aligned}
$$
So,

$$
f_{11} \rightarrow \frac{s}{E A_{\xi}}, \quad f_{12} \rightarrow 0, \quad f_{16} \rightarrow 0, \quad f_{22} \rightarrow \frac{s^{3}}{3 E I_{\zeta}}+\frac{s}{G A_{\eta}}, \quad f_{26} \rightarrow \frac{s^{2}}{2 E I_{\zeta}}, \quad f_{33} \rightarrow \frac{s^{3}}{3 E I_{\zeta}}+\frac{s}{G A_{\eta}}, \quad f_{4} \rightarrow 0, \quad f_{44} \rightarrow \frac{s}{G I_{\xi}}, \quad f_{45} \rightarrow 0, \quad f_{55} \rightarrow \frac{s}{E I_{\eta}},
$$
$f_{66} \rightarrow \frac{s}{E I_{6}}$

When the radius of curvature is infinite, $f$ degrades into flexibility matrix of cantilever straight beam element and $s$ is similar to the axis length of straight beam.

\section{Element Stiffness matrix of variable curvature curved beam in local coordinate system}

Make $K_{i j}=f^{-1}$, the equation can be expressed as

$$
F_{j}^{e}=K_{\ddot{j}} \delta_{j}^{e} .
$$

Relevant scholars ${ }^{[6]}$ have proved that the element stiffness matrix of constant curvature curved beam in local coordinate system can be expressed as

$$
K^{e}=\left[\begin{array}{cc}
H K_{i j} H^{T} & H K_{j j} \\
K_{j j} H^{T} & K_{j j}
\end{array}\right]
$$

In a similar way, the element stiffness matrix of variable curvature curved beam in local coordinate system can also be expressed as the above equation. " $H$ " is the expression of balance matrix (9-10) at end $i$ which is expressed in the beginning.

\section{Conclusion}

In this paper, starting from the basic ideas of structural matrix analysis, the stiffness matrix and equivalent node loads of space curvature curved beam element are obtained. The calculation 
formulas are expressed in the form of matrix, which can achieve the application in the computer programming. All coefficients in the paper can be directly referenced.

Because the equivalent node loads of variable curvature curved beam element which are caused by any space force system are obtained. In this way, the structural calculation of variable curvature curved beam becomes easy.

The finite element method of constant curvature curved beam which is well known by us is only a special case when the study result of this paper is with the parameter $b=0$. Thus its scope of application will be improved.

\section{Reference}

[1] Martinelli, E., C. Faella and D. G. Palma: Shear-Flexible Steel-Concrete Composite Beams in Partial Interaction: Closed-Form "Exact" Expression of the Stiffness Matrix( Eng. Mech,U.S.A.2012), 138(2), 151-163.

[2] Rahami, H., A. Kaveh and I. Shojaei: Analysis of Irregular Structures Composed of Regular and Irregular Parts Using Graph Products( Comput. Civ. Eng, U.S.A.2014)

[3] López-Reyes, P., A. Lorenzana and Cacho-Pérez: Limit Analysis of Frames Based on the Navier-Bernoulli Beam Model with Local Plasticity, Damage, and Hardening (Eng. Mech,U.S.A.2014)

[4] Nevling, D.: Linzell Curved Girder Deformation Prediction Effectiveness Using First-Order, Linear Geometric Finite-Element Models (Pract. Period. Struct. Des. Constr., U.S.A.2012).

[5] Tsavdaridis, K. and C. D. Mello:Vierendeel Bending Study of Perforated Steel Beams with Various Novel Web Opening Shapes through Nonlinear Finite-Element Analyses (Struct. Eng.,U.S.A.2012)

[6]Mohamadi-Shooreh, M., M. Mofid, and S. McCabe, Empirical Model of the Moment-Rotation Curve of Beam-to-Beam Bolted Flush Endplate Connections (Struct. Eng., U.S.A.2012)

[7] Sharafbayani, M. and D. Linzell: Optimizing Horizontally Curved, Steel Bridge, Cross-Frame Arrangements to Enhance Construction Performance (Bridge Eng., U.S.A.2014)

[8] Khalafalla, I. and K. Sennah, Curvature Limitations for Slab-on-I-Girder Bridges (Bridge Eng., U.S.A.2014)

[9] Xu, L., X. Lu, H. Guan and Y. Zhang: Finite-Element and Simplified Models for Collision Simulation between Overheight Trucks and Bridge Superstructures (Bridge Eng., U.S.A.2013)

[10] Davids, W., T. Poulin and K. Goslin: Finite-Element Analysis and Load Rating of Flat Slab Concrete Bridges (Bridge Eng., U.S.A.2013)

[11] Wu, H. Q. , in: Finite element analysis of structure, edtied by Chinese Railway Press (2000)

[12] Tong, G., and Q. Xu, in: Linear and nonlinear analysis theory of thin-walled curved box girders, edtied by Scientific Press(2004)

[13] Fu, Y.: The distance formula and application between point and straight line in polar coordinates (Shangrao Teachers College Journal,China1998) 